\title{
NMR Detection of an Equilibrium Phase Consisting of Monomers and Clusters in Concentrated Lysozyme Solutions
}

\author{
Suliman Barhoum ${ }^{\dagger}$ and Anand Yethiraj* \\ Department of Physics and Physical Oceanography, Memorial University, St. John's, \\ Newfoundland and Labrador, Canada \\ Received: September 20, 2010; Revised Manuscript Received: November 15, 2010
}

\begin{abstract}
Protein aggregation is an important biophysical phenomenon, and it is technically challenging to quantify. Scattering studies in concentrated protein solutions are not in complete agreement over the existence of an equilibrium cluster phase. We use pulsed-field-gradient NMR spectroscopy to characterize diffusion in the long-time limit in concentrated lysozyme solutions and find strong evidence for the existence of an equilibrium phase that consists of both lysozyme monomers and clusters (aggregates). They indicate too that there is rapid exchange between monomer and aggregate on the NMR time scale, and that macroscopic measurables (e.g., the relaxation rate and the observed diffusion coefficient) reflect a weighted average of the two fractions. Our results are quantitatively compared, with no fit parameters, to simple theories of macromolecular crowding.
\end{abstract}

\section{Introduction}

The interactions of proteins in water is important in many biophysical processes. Concentrated solutions of protein exhibit a tendency to self-associate to form aggregates. ${ }^{1}$ This phenomenon is important because many human proteins have segments that can form amyloid aggregates, ${ }^{2}$ which are implicated in the root causes of many "conformational diseases". 3,4 On the other hand, much can be learned about structure and conformations of proteins in solution by treating them as polymeric colloidal suspensions, ${ }^{5}$ with a phase behavior that includes liquid-liquid phase separation ${ }^{6}$ and the formation of crystalline aggregates. ${ }^{7,8}$ Dynamics in concentrated protein solutions is also complicated by effects of macromolecular crowding. ${ }^{9,1}$ This too can be modeled in analogy with behavior in dense colloidal suspensions. ${ }^{10,11}$

For this reason, the aggregation of lysozyme solutions has been studied extensively using scattering techniques, ${ }^{12-18}$ nuclear magnetic resonance, ${ }^{7,8,19,16}$ and computer simulations. ${ }^{20,21}$ Lysozyme solutions in the concentrated regime have been shown to exhibit metastable liquid-liquid phase segregation ${ }^{6,22}$ that is in some cases followed by crystallization and precipitation. . $7,19,18$ Aggregates detected in solution in the latter studies are transient aggregates.

However, small-angle scattering studies have reported too that concentrated lysozyme solutions form not only transient aggregates but also a stable equilibrium phase consisting of both individual proteins (referred to here as "monomers") and clusters. ${ }^{12,13}$ Parameters like temperature, salt concentration, and $\mathrm{pH}$ were found to play a role in changing cluster size and cluster density. ${ }^{12,13}$ These results provide support for a colloidal model to understand the phase behavior of lysozyme, ${ }^{23}$ with the generic feature of long-range repulsions and short-range attractions yielding the possibility for complex gel and glass phases in lysozyme solutions. ${ }^{24}$

The small-angle scattering evidence for equilibrium clusters is based on one interpretation of the interference peak observed at low scattering vectors. A second small-angle scattering study, ${ }^{14}$ which also observes an interference peak, however,

\footnotetext{
* To whom correspondence should be addressed. E-mail: ayethiraj@ mun.ca.
}

†E-mail: sulimanb@mun.ca. attributes it to interactions of individual monomers. In addition, a recent small-angle neutron scattering and neutron spin echo study has proposed a scenario that invokes the existence of clusters ("dynamic clusters") but with macroscopic properties in the long time limit that are determined by monomeric proteins. ${ }^{15}$ Thus scattering studies ${ }^{12,14,15,25}$ are still not in complete agreement on the existence of an equilibrium cluster phase.

In this context, we present an NMR study in concentrated lysozyme solutions that provides quantitative and independent evidence, in the long time limit, for an equilibrium phase that consists of fractions of protein monomers and fractions of aggregates or clusters. We find too that proteins exchange rapidly between monomer and cluster form on the NMR time scale. The NMR signal is a weighted average of the different fractions, and the macroscopic properties are governed by this weighted average. Monomer diffusion coefficients are compared with theoretical models for long-time self-diffusion in crowded solutions: good agreement is found with the model of Han and Herzfeld. ${ }^{10}$

\section{Experimental Section}

Hen egg white lysozyme with 14600 average molecular mass and HEPES buffer were purchased from Sigma-Aldrich Canada. We prepared $20 \mathrm{mM}$ HEPES buffer solution in $\mathrm{D}_{2} \mathrm{O}$ at $\mathrm{pH} \approx$ 7.5 and used it to prepare lysozyme stock solution with $40 \mathrm{mg}$ / $\mathrm{mL}$ lysozyme concentration. The lysozyme stock solution was stirred and filtered using a $0.22 \mu \mathrm{m}$ filter to remove undissolved material. A centrifugal filter device with a YM-10 membrane was used to concentrate the lysozyme stock solution more. This high concentration lysozyme stock solution was diluted with the $20 \mathrm{mM}$ HEPES buffer solution to prepare the lysozyme samples (batch 1, prepared from $274 \mathrm{mg} / \mathrm{mL}$ stock solution: 14 $\mathrm{mg} / \mathrm{mL}, 36 \mathrm{mg} / \mathrm{mL}, 169 \mathrm{mg} / \mathrm{mL}$, and $245 \mathrm{mg} / \mathrm{mL}$; batch 2, prepared from $247 \mathrm{mg} / \mathrm{mL}$ stock solution: $70 \mathrm{mg} / \mathrm{mL}$ and 100 $\mathrm{mg} / \mathrm{mL}$; batch 3, prepared from $274 \mathrm{mg} / \mathrm{mL}$ stock solution: 93 $\mathrm{mg} / \mathrm{mL}$ ). The lysozyme concentration was measured after preparation via the absorbance at $\lambda=595 \mathrm{~nm}^{26}$ using a UV-visible spectrometer. In increasing order, these concentrations resulted in lysozyme volume fractions of $\Phi=0.012$, $0.031,0.059,0.079,0.085,0.143$, and 0.215 . 


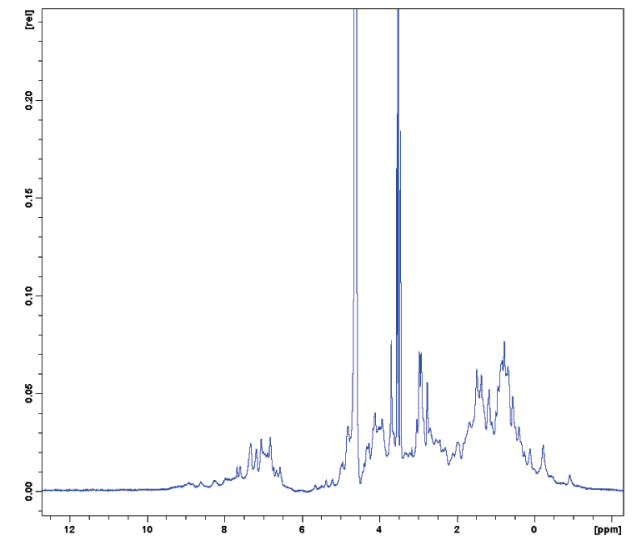

(a)

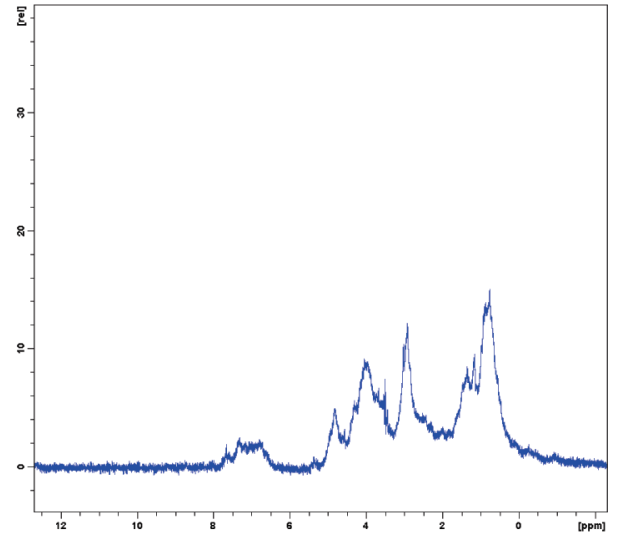

(b)

Figure 1. Lysozyme $(254 \mathrm{mg} / \mathrm{mL}) / \mathrm{HEPES} / \mathrm{D}_{2} \mathrm{O}$, sample temperature $298 \mathrm{~K}$. (a) ${ }^{1} \mathrm{H}$ NMR one-dimensional (1D) spectrum of sample. (b) ${ }^{1} \mathrm{H}$ NMR $1 \mathrm{D}$ spectrum after a pulsed-gradient stimulated echo $(\Delta=100 \mathrm{~ms}$, gradient strength $=0.62 \mathrm{~T} / \mathrm{m})$.

Figure 1a shows the one-dimensional NMR spectrum of the highest lysozyme concentration sample $C=254 \mathrm{mg} / \mathrm{mL}$ in an aqueous solution, while Figure $1 \mathrm{~b}$ shows the Fourier transform of a pulsed gradient stimulated echo at a gradient strength where the water and HEPES signals are completely attenuated, leaving only the lysozyme spectrum. NMR experiments were carried out on a $600 \mathrm{MHz}$ Bruker Avance II spectrometer equipped with a Micro-5 imaging (3-axis gradient) probe with a maximum gradient strength of $200 \mathrm{G} / \mathrm{cm}(2 \mathrm{~T} / \mathrm{m})$. Unless otherwise stated, all NMR experiments were performed at $T=298 \mathrm{~K}$.

The self-diffusion results were obtained with a pulsed field gradient stimulated-echo sequence with (almost square) trapezoidal gradient pulses. The diffusion coefficient of the lysozyme species in aqueous solution is obtained from the attenuation of the signal: ${ }^{27}$

$$
\ln \left(\frac{S(k)}{S(0)}\right)=-D k
$$

where $S(k)$ is the "intensity" of the signal (the integration of the relevant peak region) in the presence of the field gradient pulse, $S(0)$ is the intensity of the signal in the absence of field gradient pulse, $k=(\gamma \delta \mathrm{g})^{2}(\Delta-\delta / 3)$ is a generalized gradient strength parameter, $g$ is the amplitude of the field gradient pulse, $\gamma=\gamma^{\mathrm{H}}=2.6571 \times 10^{8} \mathrm{~T}^{-1} \cdot \mathrm{s}^{-1}$ is the gyromagnetic ratio of the ${ }^{1} \mathrm{H}$ nucleus, $\delta=2 \mathrm{~ms}$ is the duration of the field gradient pulse, and $\Delta$ is the time period between the two field gradient pulses which was varied from 50 to $700 \mathrm{~ms}$.

The relaxation measurements were also performed using the imaging probe. The inversion recovery technique with 16 time delays $t$ was used to measure $T_{1}$, and the intensity data of lysozyme peaks in the region between 6.5 and $9.5 \mathrm{ppm}$ were fit to the equation $I(T)=I_{\mathrm{O}}\left(1-2 \exp \left(-t / T_{1}\right)\right)$. A series of $16(\pi /$ $2)_{x}-t / 2-(\pi)_{x}-t / 2-$ acquire spin echo experiments with varying delay times $t$ was used to measure $T_{2}$, obtained similarly from a fit of the intensities to the equation $I(t)=I_{\mathrm{O}} \exp \left(-t / T_{2}\right)$.

\section{Results and Discussion}

Crowded Diffusion. Figure 2 a shows a multiexponential attenuation in the signal that is due to the existence of water and HEPES molecules in the solution in addition to lysozyme species. The early linear (small $k$ ) and later linear (large $k$ ) parts in the signal attenuation have distinct spectral signatures that overlap in chemical shift but are separable in the field-gradient dimension (Figure 1). The linear regime at large $k$ corresponds to the signal attenuation associated with lysozyme peaks in the spectrum, which is monoexponential. This is consistent also with relaxation results where the time dependence of the lysozyme signal attenuation exhibits a single relaxation time.

Figure $2 \mathrm{~b}$ shows the variation of the lysozyme self-diffusion coefficient $D$ at diffusion time $\Delta=50,100$, and $150 \mathrm{~ms}$ as a function of lysozyme volume fraction:

$$
\Phi=C \times\left(\frac{N_{\mathrm{A}} \times\left(\frac{4 \pi R^{3}}{3}\right)}{M_{\mathrm{w}}}\right) \times 10^{3} \approx C \times 0.85 \times 10^{-3}
$$

treating the globular protein lysozyme as an ellipsoid with dimensions $a=b=15 \AA$ and $c=22.5 \AA$, ${ }^{31}$ leading to hydrodynamic radius $R=(a b c)^{1 / 3} \approx 17 \AA$. In eq $2 N_{\mathrm{A}}$ is the Avogadro number and $M_{\mathrm{W}}$ is the molecular weight of lysozyme. If one used instead the specific volume of lysozyme, ${ }^{32}$ one gets a lower value of volume fraction $\Phi=C \times 0.717 \times 10^{-3}$. The uncertainty in volume fraction reflects a systematic difference in the calculation of volume fraction based on the bare lysozyme volume (lower value) and the volume fraction deduced from the hydrodynamic radius of the lysozyme molecule. The diffusion coefficients are scaled by the theoretical value at zero concentration $D_{0}=11.7 \times 10^{-11} \mathrm{~m}^{2} / \mathrm{s}$, ${ }^{19}$ which employs an anisotropy correction to the calculation for spheres. ${ }^{28}$

Figure $2 \mathrm{~b}$ shows that the lysozyme self-diffusion coefficient $D$ increases with decreasing lysozyme volume fraction $\Phi$. Also plotted are the long-time self-diffusion coefficient according to Tokuyama and Oppenheim, ${ }^{11}$ Medina-Noyola ${ }^{29}$ (using the formula obtained in van Blaaderen et al. ${ }^{30}$ ), and Han and Herzfeld ${ }^{10}$ (using the self-consistent value $\Delta r / R=$ $2 / 3$ ); we would expect the experiments to be closest to the model values for low volume fractions of lysozyme in solution where aggregate formation is least important. For $\Phi<0.1$, the Medina-Noyola form appears to be closest to the observed diffusion coefficient results for lysozyme. The Medina-Noyola and Tokuyama-Oppenheim theoretical curves underestimate the diffusion coefficients at higher volume fractions; this fact has been noted before ${ }^{19}$ and can be understood simply as follows:

- The observed diffusion coefficient is a relaxation-weighted average of monomer and aggregate diffusion, as expressed 


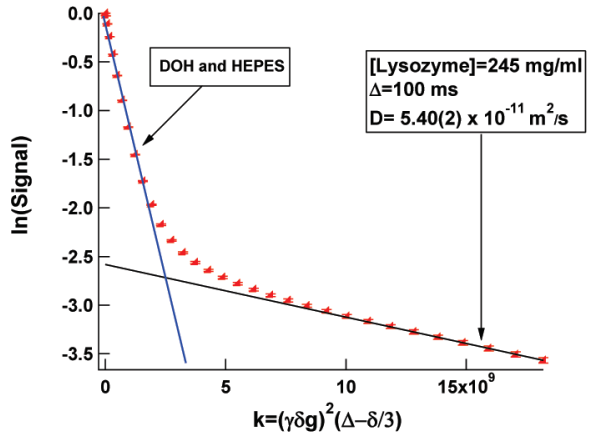

(a)

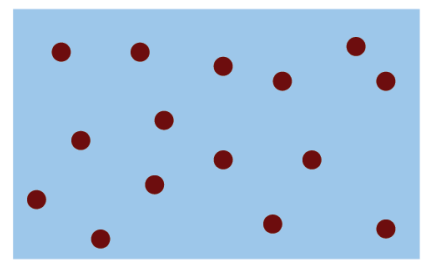

monodisperse

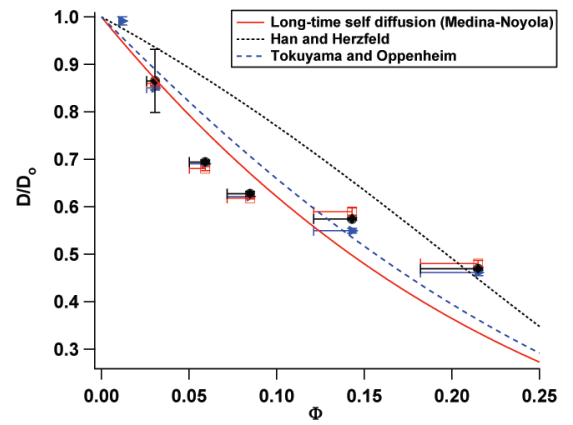

(b)

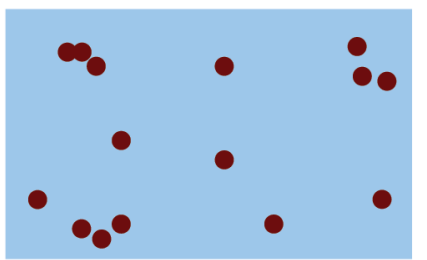

monomers+aggregates

\section{(c)}

Figure 2. (a) Attenuation of the signal $S(k) / S(0)$ on a log scale versus $K=(\gamma \delta g)^{2}(\Delta-\delta / 3)$ for $254 \mathrm{mg} / \mathrm{mL}(\Phi=0.215)$ lysozyme concentration and with $\Delta=100 \mathrm{~ms}$. The black line shows the attenuation in signal of lysozyme peaks. The blue line shows the attenuation in signal of DOH and HEPES peaks. (b) Lysozyme self-diffusion coefficient $D$ (scaled by the theoretical value at zero concentration ${ }^{28,19}$ at diffusion times $\Delta=50,100$, and $150 \mathrm{~ms}$ versus lysozyme volume fraction $\Phi$. Dashed lines are long-time self-diffusion coefficients obtained in the works of Medina-Noyola, ${ }^{29,30}$ Tokuyama and Oppenheim, ${ }^{11}$ and Han and Herzfeld. ${ }^{10}$ Volume fraction error bars (left error bars). (c) Illustration of the effect of aggregate formation on the free volume seen by monomers.

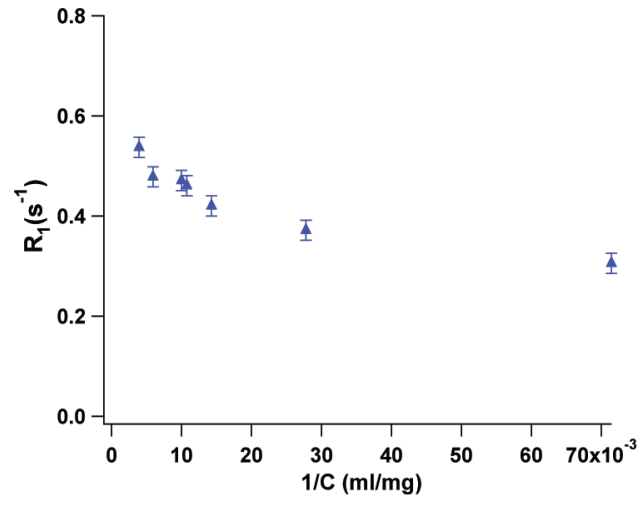

(a)

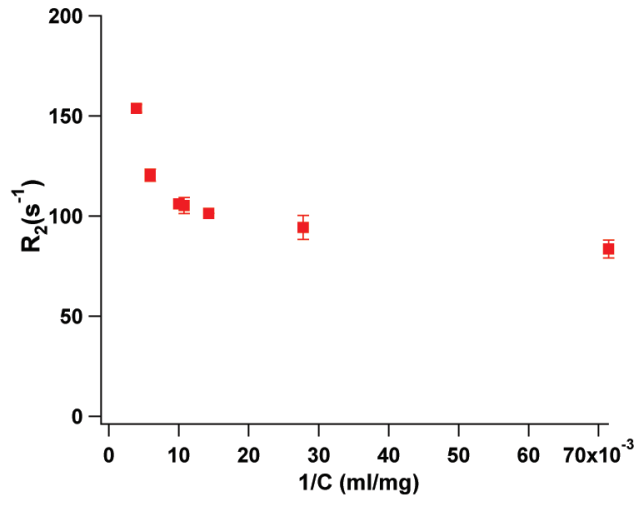

(b)

Figure 3. (a) Proton longitudinal relaxation rates $R_{1}$ and (b) transverse relaxation rates $R_{2}$ versus reciprocal of lysozyme concentration $1 / C$. Both relaxation rates show a systematic decrease as a function of $1 / C$.

in eq 4. This alone would indicate that the observed diffusion coefficient is a lower bound for the monomer diffusion.

- When there are aggregates, the calculated volume fraction reflects an underestimation of the free volume and an overestimation of the effective volume fraction seen by the monomers. This latter point is illustrated in the cartoon (Figure 2c).

We revisit the comparison of experimental diffusion coefficients with theory after a more careful analysis of the effects of relaxation weighting on the observed diffusion coefficients.

Relaxation Rates. Parts $\mathrm{a}$ and $\mathrm{b}$ of Figure 3 show the variation of longitudinal relaxation rate $R_{1}\left(=1 / T_{1}\right)$ and transverse relaxation rate $R_{2}\left(=1 / T_{2}\right)$, respectively, as a function of reciprocal lysozyme concentration $1 / C$. On the basis of the assumption that the lysozyme molecules coexist with lysozyme aggregates especially at high lysozyme concentration, the observed relaxation rates $R_{i}$ (i.e., $R_{1}$ and $R_{2}$ ) include contributions from both the aggregate relaxation rate $R_{i, \mathrm{a}}$ and the monomeric relaxation rate $R_{i, \mathrm{~m}}$. Therefore, the observed relaxation rates $R_{1}$ and $R_{2}$ may be written

$$
R_{i}=b R_{i, \mathrm{~m}}+(1-b) R_{i, \mathrm{a}}
$$

where $R_{i, \mathrm{~m}}$ and $R_{i, \mathrm{a}}$ are $R_{1, \mathrm{~m}}$ or $R_{2, \mathrm{~m}}$ and $R_{1, \mathrm{a}}$ or $R_{2, \mathrm{a}}, b=C_{\mathrm{m}} / C$ is the fraction of the free lysozyme monomers, and $C_{\mathrm{m}}$ is the free monomer concentration.

Parts $\mathrm{a}$ and $\mathrm{b}$ of Figure 3 are clearly not a linear function of $1 / C$ (eq 3). In simpler micelle- and aggregate-forming systems (such as SDS and PEO-SDS solutions ${ }^{33}$ ) there is a linear dependence of the relaxation rates on $1 / C$ : this implies that the concentration of free monomers is a constant above a critical aggregation concentration. In this system, the free fraction of monomers is itself a function of $C$. 


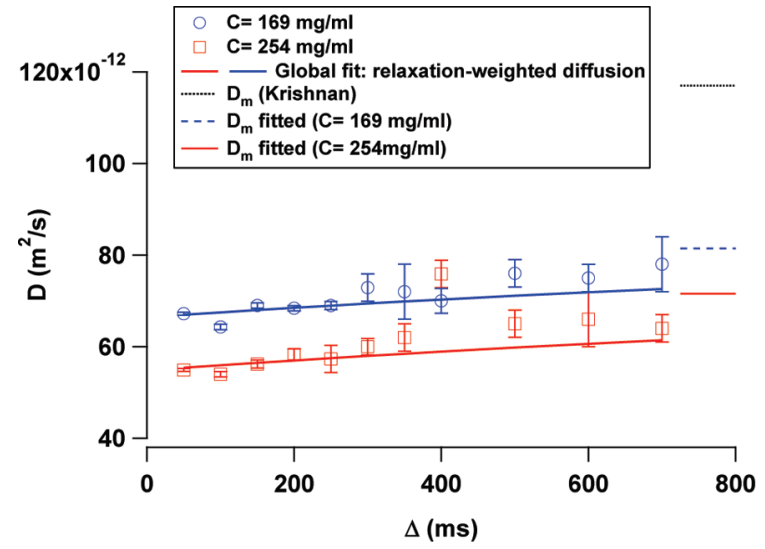

(a)

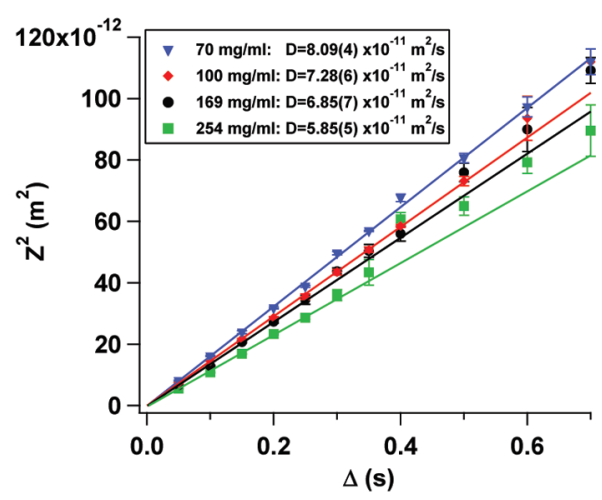

(b)

Figure 4. (a) Lysozyme self-diffusion coefficient $D$ versus diffusion time $\Delta$ for lysozyme concentrations $C=169 \mathrm{mg} / \mathrm{mL}(\Phi=0.143)$ and 254 $\mathrm{mg} / \mathrm{mL}(\Phi=0.215)$. The values of $D_{\mathrm{m}}$ obtained from the fit are shown to the right of the data. (b) Mean-squared displacement $z^{2}=2 D \Delta$ as a function of $\Delta$ for $C=70,100,169$, and $254 \mathrm{mg} / \mathrm{mL}$.

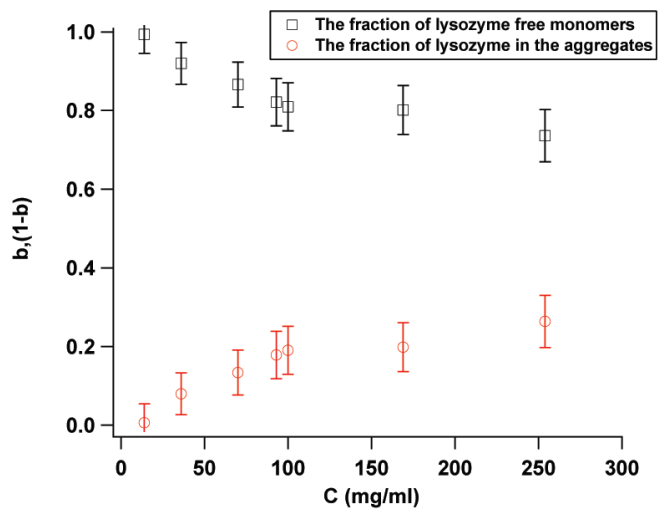

(a)

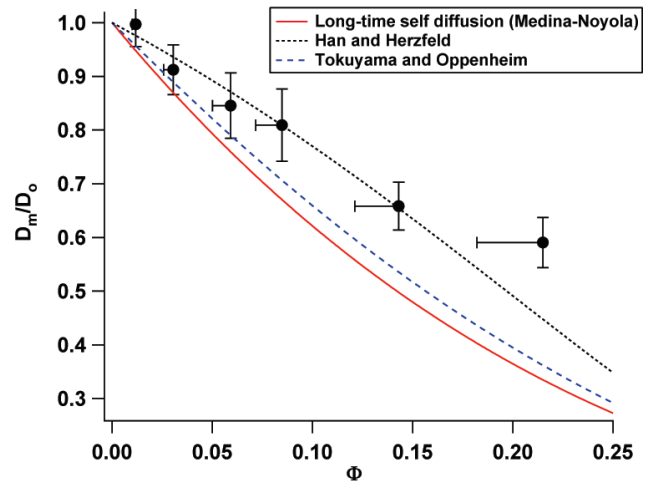

(b)

Figure 5. (a) Fraction $b$ of lysozyme free monomers and the fraction $(1-b)$ of lysozyme monomers in the aggregates versus lysozyme concentration $C$. (b) Monomeric self-diffusion coefficient $D_{\mathrm{m}} / D_{0}$ (scaled by the theoretical value at zero concentration ${ }^{19,28}$ ) at $\Delta=100 \mathrm{~ms}$ versus lysozyme volume fraction $\Phi$. Dashed lines are long-time self-diffusion coefficients obtained in the works of Medina-Noyola, ${ }^{29,30}$ Tokuyama and Oppenheim, ${ }^{11}$ and Han and Herzfeld. ${ }^{10}$

Fraction of Protein Monomers and Clusters. Figure 4a shows the variation of lysozyme self-diffusion coefficient $D$ at two different lysozyme concentrations $(C=169$ and $254 \mathrm{mg} /$ $\mathrm{mL}$ ) as a function of the diffusion time $\Delta$. At lower concentrations, we have limited signal at high $\Delta$. At all concentrations, the signal attenuation of lysozyme peaks is monoexponential over the range of diffusion time $\Delta$ probed (not shown). The lysozyme self-diffusion coefficient shows a small but unambiguous increase with diffusion time for both $C=169$ and $254 \mathrm{mg} /$ $\mathrm{mL}$.

Figure $2 b$ shows that in the time scale of the NMR experiment we are already accessing the long time selfdiffusion coefficient of lysozyme at $\Delta=50 \mathrm{~ms}$. Therefore, for a monodisperse system of lysozyme proteins we would not expect $D$ to show a dependence of $\Delta$ for $\Delta>50 \mathrm{~ms}$. In addition, if we were not in the long-time limit, we would expect to see a decrease not an increase ${ }^{34}$ However, if the lysozyme exists in monomeric form and in clusters, then these two states would be expected to have different microscopic correlation times: shorter for monomers and longer for aggregates. Then the observed diffusion coefficients from a pulsed-gradient stimulated echo would be a (longitudinal) relaxation weighted average of the monomer and the aggregate diffusion coefficient. ${ }^{19,7}$ Thus, the observed lysozyme self-diffusion coefficient $D$ can be written in the form

$$
D=\frac{b D_{\mathrm{m}} \exp \left(-R_{1, \mathrm{~m}} \Delta\right)+(1-b) D_{\mathrm{a}} \exp \left(-R_{1, \mathrm{a}} \Delta\right)}{b \exp \left(-R_{1, \mathrm{~m}} \Delta\right)+(1-b) \exp \left(-R_{1, \mathrm{a}} \Delta\right)}
$$

where from eq $3, b=\left(R_{1}-R_{1, \mathrm{a}}\right) /\left(R_{1, \mathrm{~m}}-R_{1, \mathrm{a}}\right)$. By globally fitting the diffusion curves of both $C=169$ and $254 \mathrm{mg} / \mathrm{mL}$ to eq 4 , we are able to calculate the monomer and aggregate relaxation rates $\left(R_{1, \mathrm{~m}} \approx 0.30(4) \mathrm{s}^{-1}, R_{1, \mathrm{a}} \approx 1.2(1) \mathrm{s}^{-1}\right.$, and $\left.D_{\mathrm{a}} \approx 8(8) \times 10^{-12} \mathrm{~m}^{2} / \mathrm{s}\right)$. There is a large uncertainty in $D_{\mathrm{a}}$. We can say unambiguously, however, that $D_{\mathrm{a}} \ll D_{\mathrm{m}}$. An alternative approach is to calculate the mean-squared displacement $z^{2}=2 D \Delta$ (ignoring the effects of relaxation weighting, Figure $4 \mathrm{~b}$ ). The slope in Figure $4 \mathrm{~b}$ yields a reasonable estimate (although, comparing with the fit to Figure $4 \mathrm{a}$, in fact an underestimate of about $10 \%$ at higher concentrations) of the monomer diffusion coefficient.

From the monomer and aggregate relaxation rates $\left(R_{1, \mathrm{~m}}\right.$ and $R_{1, \mathrm{a}}$ ) and the measured relaxation rates $R_{1}$, we can obtain (eq 3 ) the fraction $b$ of lysozyme free monomers in the solution and the fraction $(1-b)$ of lysozyme molecules in the aggregate state for all samples; Figure 5a shows that the fraction $b$ of free lysozyme monomers decreases by increasing the concentration, while the fraction $(1-b)$ of lysozyme monomers in the aggregate state increases with increasing concentration. At large concentrations, approximately $25 \%$ of lysozyme molecules are in the aggregate state. Using eq 4 and the calculated $R_{1, \mathrm{~m}}, R_{1, \mathrm{a}}$, 


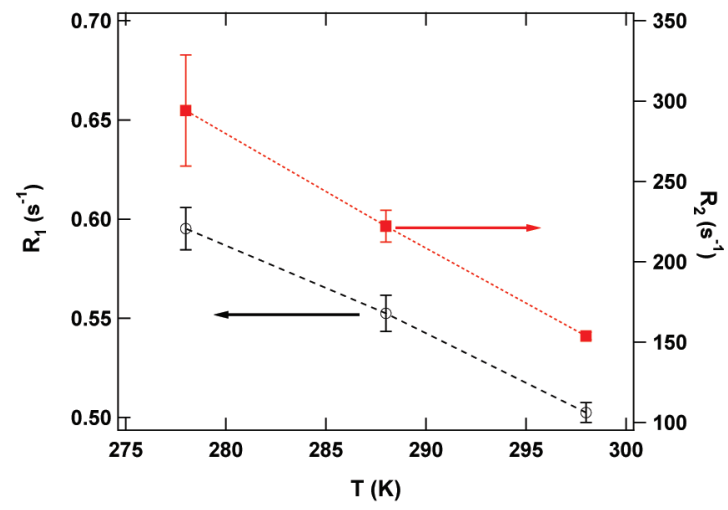

(a)

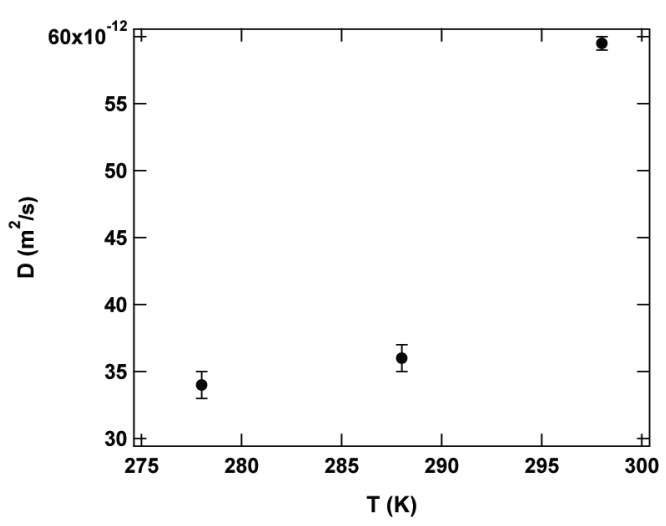

(b)

Figure 6. (a) Relaxation rates $R_{1}$ (open circles, left scale) and $R_{2}$ (filled squares, right scale) versus temperature $T$. (b) Lysozyme diffusion coefficient $D$ versus temperature. $C=254 \mathrm{mg} / \mathrm{mL}$.

and $D_{\mathrm{a}}$ at $\Delta=100 \mathrm{~ms}$, we calculate the scaled lysozyme monomeric diffusion coefficient $D_{\mathrm{m}} / D_{0}$ at different lysozyme volume fractions $\Phi$ (Figure $5 \mathrm{~b}$ ). A rough estimate of the error in $D_{\mathrm{m}} / D_{0}$ is obtained from the errors in the global fitting. The $\Phi$ dependence of the monomer diffusion coefficients exhibit reasonable agreement with the theoretical results of Han and Herzfeld up to $\Phi \approx 0.15$.

From the fit to the diffusometry results, we can plot the longitudinal relaxation rate $R_{1}$ and transverse relaxation rate $R_{2}$, respectively, as a function of the fraction $b$ of free lysozyme monomers (not shown). By fitting both curves to eq 3 , we are able to calculate the longitudinal and transverse relaxation rates of lysozyme monomer and aggregate. While $R_{1, \mathrm{~m}}=0.29(7)$ $\mathrm{s}^{-1}$ and $R_{1, \mathrm{a}}=1.2(3) \mathrm{s}^{-1}$ (for the monomer and aggregate) are simply restating the results of the global fitting in eq 4 , we are also able to obtain the corresponding tranverse relaxation rates $\left(R_{2, \mathrm{~m}}=75(6) \mathrm{s}^{-1}\right.$ and $\left.R_{2, \mathrm{a}}=325(25) \mathrm{s}^{-1}\right)$.

The relaxation rate and the diffusion measurements for 254 $\mathrm{mg} / \mathrm{mL}$ lysozyme sample have been done at three different temperatures 298, 288, and 278 K. Parts a and b of Figure 6 show the variation of longitudinal $R_{1}$ relaxation rate, transverse $R_{2}$ relaxation rate, and the diffusion coefficient, respectively, as a function of temperature. The relaxation rates $R_{1}$ and $R_{2}$ increase as the temperature decreases, while the diffusion coefficient shows a decrease as the temperature decreases. This too is consistent with the fraction of lysozyme aggregate increasing with decreasing lysozyme solution temperature.

\section{Conclusion}

We have carried out NMR relaxometry and diffusometry experiments on concentrated lysozyme solutions. Monoexponential dependence of the NMR signal on the delay time in the inversion recovery and spin echo experiments suggest that, if there are multiple species in solution they must be exchanging rapidly on the NMR time scale. Both longitudinal and transverse relaxation rates show a sharp increase with increasing concentration, suggesting the increased contribution to the signal from lysozyme clusters or aggregates.

Diffusometry as a function of lysozyme volume fraction $\Phi$ exhibits agreement with simple colloidal models for macromolecular crowding for $\Phi<0.1$, with no fit parameters. At higher volume fractions or concentrations, there is a marked deviation, again pointing to the role of aggregates. All the models underestimate the observed diffusion coefficients for large $\Phi$. A simple picture for this is that the formation of aggregates creates more free volume for the monomers.
The dependence of diffusion coefficient on diffusion time $\Delta$ provides quantitative evidence of aggregates. Prior to approaching the long-time self-diffusion limit, one would expect a decrease in $D$ with increasing $\Delta$. Given that the experiments are clearly in the long-time limit $\left(\tau_{\text {Brownian }} \sim 40 \mathrm{~ns}<<\Delta\right.$ ), one would naively expect no $\Delta$ dependence for a monodisperse system. The presence of aggregates (with a relaxation rate larger than that of monomers) results in the observed diffusion coefficients being a relaxation-weighted average of monomer and aggregate diffusion. Unique to NMR is the fact that microscopic molecular correlation times in the picosecond to nanosecond window lead to relaxation times in the millisecond to second window, where one is probing long-time selfdiffusion. Putting together both diffusion measurements (e.g., Figure 4a) and relaxation measurements, we were able to calculate the relaxation rates of lysozyme in the monomer and the aggregate state.

In addition, we obtained from the fit in Figure $4 \mathrm{a}$ the selfdiffusion coefficient of lysozyme monomers $D_{\mathrm{m}}$ as a function of volume fraction, as well as of the aggregates $D_{\text {a }}$. The monomeric diffusion coefficient is in rather good agreement with simulations of crowded diffusion of model proteins (Han and Herzfeld $^{10}$ ). The value obtained for aggregate diffusion is $D_{\mathrm{a}} \approx$ $8 \times 10^{-12} \mathrm{~m}^{2} / \mathrm{s}$. If the clusters were compact and freely diffusing, this implies an aggregate size of $9 R_{0}$, about 2-3 times larger than the values deduced by Porcar et al. ${ }^{15}$ and Stradner et al. ${ }^{12}$ A possibility that is consistent with our experiments and with the picture of an equilibrium cluster phase driven by competition between short-range attraction and long-range repulsion (as suggested by Stradner et al. ${ }^{12}$ ) is that the clusters are not tightly bound oligomers but instead form loose percolating networks at higher density coexisting with, and exchanging with, monomers at lower densities. This has been observed in colloidal systems. ${ }^{35}$ Finally, we quantified (Figure 5a) the fraction $b$ and $1-b$ of free monomers and aggregates over the entire range of lysozyme concentration. Above $C=100 \mathrm{mg} / \mathrm{mL}$ (at $T=$ $298 \mathrm{~K}$ ), the aggregate state constitutes a fraction of roughly $20-25 \%$ of the proteins in solution; this fraction increases with decreasing $T$. The macroscopic properties of the long-time limit are therefore governed by a weighted average of monomeric and aggregate properties.

Acknowledgment. We thank the Natural Sciences and Engineering Research Council (NSERC) of Canada for financial support of this work. We acknowledge discussions with and 
technical help from Dr. Celine Schneider and the help of Ms. Donna Jackman in lysozyme sample preparation.

\section{References and Notes}

(1) White, D. A.; Buell, A. K.; Knowles, T. P. J.; Welland, M. E.; Dobson, C. M. J. Am. Chem. Soc. 2010, 132, 5170-5175.

(2) Schnabel, J. Nature 2010, 464, 828-829.

(3) Kopito, R. R.; Ron, D. Nat. Cell Biol. 2000, 2, E207-E209. 248 .

(4) Aguzzi, A.; O'Connor, T. Nat. Rev. Drug Discovery 2010, 9, $237-$

(5) Elcock, A. H.; Sept, D.; McCammon, J. A. J. Phys. Chem. B 2001, 105, 1504-1518.

(6) Muschol, M.; Rosenberger, F. J. Chem. Phys. 1997, 107, 19531962.

(7) Price, W. S.; Tsuchiya, F.; Arata, Y. Biophys. J. 2001, 80, 15851590 .

(8) Ilyina, E.; Roongta, V.; Pan, H.; Woodward, C.; Mayo, K. H. Biochemistry 1997, 36, 3383-3388.

(9) Minton, A. P. Curr. Opin. Struct. Biol. 2000, 10, 34-39.

(10) Han, J.; Herzfeld, J. Biophys. J. 1993, 65, 1155-1161.

(11) Tokuyama, M.; Oppenheim, I. Phys. Rev. E 1994, 50, R16-R19.

(12) Stradner, A.; Sedgwick, H.; Cardinaux, F.; Poon, W. C. K.; Egelhaaf, S. U.; Schurtenberger, P. Nature 2004, 432, 492-495.

(13) Stradner, A.; Cardinaux, F.; Schurtenberger, P. J. Phys. Chem. B 2006, 110, 21222-21231.

(14) Shukla, A.; Mylonas, E.; Cola, E. D.; Finet, S.; Timmins, P.; Narayanan, T.; Svergun, D. I. Proc. Natl. Acad. Sci. U. S. A. 2008, 105, 5075-5080.

(15) Porcar, L.; Falus, P.; Chen, W.-R.; Faraone, A.; Fratini, E.; Hong, K.; Baglioni, P.; Liu, Y. J. Phys. Chem. Lett. 2009, 1, 126129.

(16) Poznanski, J.; Szymanski, J.; Basinska, T.; Slomkowski, S.; Zielenkiewicz, W. J. Mol. Liq. 2005, 121, 21-26.
(17) Liu, Y.; Fratini, E.; Baglioni, P.; Chen, W.-R.; Chen, S.-H. Phys. Rev. Lett. 2005, 95, 1-4.

(18) Niimura, N.; Minezaki, Y.; Ataka, M.; Katsura, T. Cryst. Growth 1995, 154, 136-144.

(19) Price, W. S.; Tsuchiya, F.; Arata, Y. J. Am. Chem. Soc. 1999, 121, $11503-11512$.

(20) McGuffee, S. R.; Elcock, A. H. J. Am. Chem. Soc. 2006, 128, $12098-12110$

(21) Carlsson, F.; Malmsten, M.; Linse, P. J. Phys. Chem. B 2001, 105, 12189-12195.

(22) Taratuta, V. G.; Holschbach, A.; Thurston, G. M.; Blankschtein, D.; Benedek, G. B. J. Phys. Chem. 1990, 94, 2140-2144.

(23) Gögelein, C.; Nägele, G.; Tuinier, R.; Gibaud, T.; Stradner, A.; Schurtenberger, P. J. Chem. Phys. 2008, 129, 085102.

(24) Cardinaux, F.; Gibaud, T.; Stradner, A.; Schurtenberger, P. Phys. Rev. Lett. 2007, 99, 118301.

(25) Trewhella, J. Proc. Natl. Acad. Sci. U. S. A. 2008, 105, 49674968.

(26) Bradford, M. M. Anal. Biochem. 1976, 72, 248-254.

(27) Price, W. S. Concepts Magn. Reson. 1997, 9, 299-336.

(28) Krishnan, V. V. J. Magn. Reson. 1997, 124, 468-473.

(29) Medina-Noyola, M. Phys. Rev. Lett. 1988, 60, 2705.

(30) van Blaaderen, A.; Peetermans, J.; Maret, G.; Dhont, J. K. G. J. Chem. Phys. 1992, 96, 4591-4603.

(31) Voet, D.; Voet, J. G.; Pratt, C. W. Fundamentals of Biochemistry: Life at the Molecular Level, 3rd ed.; John Wiley \& Sons, Inc.: New York, NY, U.S., 2008.

(32) Gekko, K.; Noguchi, H. J. Phys. Chem. 1979, 83, 2706-2714.

(33) Barhoum, S.; Yethiraj, A. J. Chem. Phys. 2010, 132, 024909.

(34) Palit, S.; Yethiraj, A. Langmuir 2008, 24, 3747-3751.

(35) Agarwal, A. K.; Yethiraj, A. Phys. Rev. Lett. 2009, 102, 198301.

JP108995K 